\section{Immune-Related Adverse Events (irAEs): Implications for Immune Checkpoint Inhibitor Therapy}

\author{
Nanruoyi Zhou, BA*; Maria A. Velez, MD*; \\ Dwight Owen, $\mathrm{MD}^{+}$; and Aaron E. Lisberg, $\mathrm{MD}^{+}$
}

$I^{2}$ mmune checkpoint inhibitors $(\mathrm{ICls})$ have revolutionized the treatment of advanced malignancies, including non-small cell lung cancer (NSCLC). Although these agents have improved clinical outcomes in NSCLC, they have also led to unique toxicities, termed immune-related adverse events (irAEs). ${ }^{1-3}$ irAEs are the result of nonspecific immune activation and can affect any organ system, although they most commonly occur in the skin, liver, gastrointestinal tract, and endocrine systems. ${ }^{4}$

In this issue of JNCCN, Kichenadasse et al ${ }^{5}$ evaluate the incidence of irAEs occurring in a group of 1,548 patients with NSCLC treated with atezolizumab monotherapy on 4 clinical trials. ${ }^{6-9}$ The authors used individual patient data from these trials to explore the incidence and timing of single-organ and multiorgan irAEs, the latter defined as either concurrent or sequential depending on whether irAEs arose within 7 days of one another. The primary objectives were to evaluate the incidence, grade, and time course of irAEs, in addition to assessing correlation with clinical outcomes (overall and progression-free survival). Kichenadasse et $\mathrm{al}^{5}$ also conducted an in-depth evaluation of multiorgan irAEs, which is of particular interest because information regarding these types of AEs is currently sparse.

Among the 4 atezolizumab NSCLC trials evaluated, multiorgan irAEs occurred in $5.4 \%$ of patients. To date, very little information is available on the frequency, significance, and management of multiorgan irAEs. Studies that have reported frequency of multiorgan irAEs have cited incidences from $5 \%$ to $43 \%$ across multiple tumor types, encompassing both single-agent and multiagent $\mathrm{ICl}$ treatment modalities. ${ }^{10-18}$ Interestingly, previously published reports have shown that the development of irAEs correlates with patient response rate and survival. ${ }^{19,20}$ In a 2019 study, Cortellini et $\mathrm{al}^{12}$ found that overall survival, progression-free survival, and objective response rates were higher among patients with NSCLC who experienced irAEs during treatment with anti-PD-1 agents; however, no significant difference in these endpoints was observed when comparing single-site versus multiple-site irAEs. In contrast, Kichenadasse et $\mathrm{al}^{5}$ found that multiorgan involvement was associated with better survival outcomes than were observed with single-organ involvement.

The question of whether multiorgan irAEs are associated with improved survival in patients with NSCLC is important because this information could potentially help guide decisions about whether to continue treatment with $\mathrm{ICl}$ s in the face of treatment-related toxicity. If multiorgan irAEs are truly associated with improved survival outcomes, clinicians and patients may choose to forge ahead with $\mathrm{ICl}$ treatment despite multiorgan toxicity, when possible. To assist with this critical decision, effective tools are needed to predict which patients are most likely to experience serious and/or fatal toxicities. Currently, however, no refined tools are available to predict development of $\mathrm{ICl}$-induced irAEs, and surveillance for occurrence generally relies on traditional laboratory markers. ${ }^{21}$ At the very least, developing predictive tools would allow for more relevant discussion between clinicians and patients during $\mathrm{ICl}$ therapy. Given the dramatic improvement in

See page 1191 for related article.

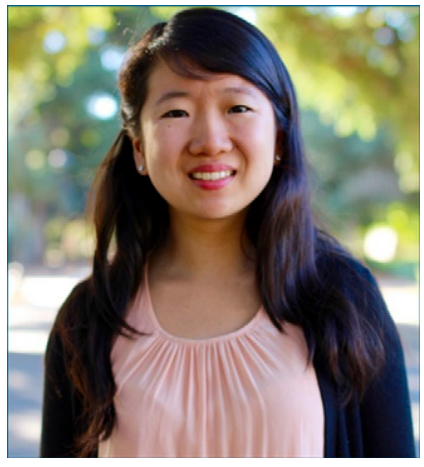

NANRUOYI ZHOU, BA

Nanruoyi (Zoe) Zhou, BA, is a third-year medical student at the David Geffen School of Medicine at UCLA. Since the beginning of her first year of medical school, she has been conducting research on immunotherapy with a focus on immune-related adverse events arising with treatment using immune checkpoint inhibitors. Although she is still undecided about what specialty to pursue in the future, she hopes to one day turn her passion for medical education and research into a fulfilling career in academic medicine.

*These authors contributed equally to this work and are listed as co-first authors.

tThese authors contributed equally to this work and are listed as co-last authors.

doi: $10.6004 /$ jnccn.2020.7640

The ideas and viewpoints expressed in this commentary are those of the author and do not necessarily represent any policy, position, or program of NCCN. 


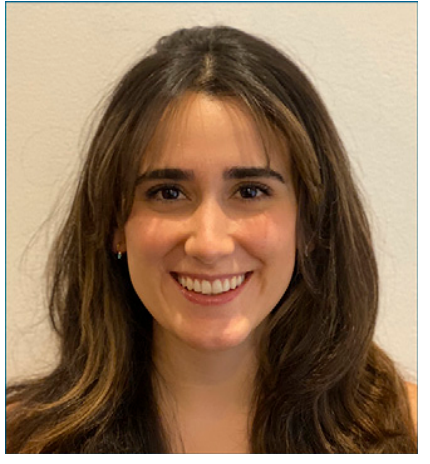

MARIA A. VELEZ, MD

Maria A. Velez, MD, is a HematologyOncology Fellow at the David Geffen School of Medicine at UCLA. Her research interests focus mainly in lung cancer, immunotherapy, and diversity inclusion in clinical trials. patient outcomes with ICls in multiple tumor types, most clinicians and patients are hesitant to defer $\mathrm{ICl}$ therapy unless treatment risk clearly outweighs benefit, which is a difficult decision point to accurately characterize.

Although irAE management guidelines exist, they are not standardized and are widely variable. In their study, Kichenadasse et al found that approximately $15 \%$ of irAEs required systemic steroids, $7.5 \%$ resulted in hospitalization, and $2.5 \%$ required treatment discontinuation. Across tumor types, anti-PD-1 discontinuation secondary to toxicity has been reported to occur at a rate of $3 \%$ to $12 \% .{ }^{22}$ Kichenadasse et al did not report the utilization rates of other immunomodulating agents, such as infliximab and tocilizumab, for the management of irAEs; however, other researchers have reported that $39.1 \%$ of patients who experienced steroid-refractory irAEs received tocilizumab. ${ }^{23}$ Although it is clear that $\mathrm{ICl}$ discontinuation secondary to irAEs occurs with some frequency, the exact incidence of this clinical outcome remains unclear, as does the optimal approach to the use of steroids and immune modulators in the management of irAEs.

Kichenadasse et al found that $27 \%$ of patients with NSCLC experienced $\geq 1$ irAEs. This result is consistent with a previous study that found that approximately $30 \%$ of patients with NSCLC treated with ICls develop irAEs. ${ }^{19}$ However, significant variability in diagnosis and attribution of irAEs was seen across the 4 atezolizumab trials evaluated, as evidenced by the fact that the observed irAE rate ranged from $7 \%$ to $40 \%$ in these trials. In the pooled analysis, most patients developed irAEs with a median time of onset of 7 weeks, but some patients developed irAEs almost 2 years after the start of treatment. The development of endocrine abnormalities in Kichenadasse et al's study (11.6\%) was lower than previously described (20\%), ${ }^{24}$ raising the question of whether these findings are secondary to undersurveillance of endocrine dysfunction or if they represent an advantage of atezolizumab over other agents with similar mechanism of action. Taken together, these findings underscore the importance of further exploring the pattern of irAE occurrence in ICl-treated advanced malignancies.

An urgent need exists to better elucidate irAEs that occur in the context of PD-1/L1 monotherapy, given the increasing use of $\mathrm{ICls}$ as part of multimodality treatment regimens, such as with chemotherapy (NSCLC: KN189), radiation (NSCLC: PACIFIC), or multikinase inhibitors (renal cell carcinoma: KN426). ${ }^{25-27} \mathrm{~A}$ better understanding of the diagnosis and management of irAEs would be especially helpful in settings where treatment modalities have overlapping toxicities, such as pneumonitis occurring after radiation or diarrhea and skin toxicities from multikinase inhibitors. Kichenadasse et al have shed additional light on a subject that is becoming increasingly common in the clinic-namely the occurrence of irAEs during $\mathrm{ICl}$ therapy-but for which treatment guidelines are still based predominantly on expert opinion and retrospective studies (eg, ASCO and NCCN guidelines). ${ }^{28,29}$

Finally, it should be noted that the patients with NSCLC included in Kichenadasse et al's meta-analysis were predominately treated in the second-line setting. ${ }^{7,8}$ As a result, some of the observed toxicity on trial, such as hematologic toxicities, may be due to prior therapies, not ICls. In fact, as the authors point out, hematologic toxicities were not considered to be related to $\mathrm{ICls}$ in these studies. However, recent studies suggest hematologic toxicities may be more common than previously thought with $\mathrm{ICls}^{30}$ When an $\mathrm{ICl}$ is combined with chemotherapy to treat advanced malignancies, it will be increasingly important to ascertain reliable attributions of these toxicities. Lack of reliable attribution may otherwise result in dose reduction or omission of chemotherapy but continued $\mathrm{ICl}$ therapy, only to result in worsening toxicity and subtherapeutic treatment.

In summary, the study by Kichenadasse et $\mathrm{al}^{5}$ in this issue of JNCCN is an important addition to our knowledge base regarding $\mathrm{ICl}$-induced irAEs, especially because this study evaluated a large atezolizumab-treated patient population from well-designed prospective studies. Undoubtedly, both single-organ and multiorgan 
irAEs will be seen with increasing frequency in clinic. Studies such as this one are a reminder of the ongoing need for research strategies to predict, detect, and mitigate toxicities from ICls.

Disclosures: Dr. Owen has disclosed that he receives grant/research support from BMS, Merck,

Palobiofarma, and Genentech. Dr. Lisberg has disclosed that he has received grant/research support from Daiichi Sankyo, Calithera Biosciences, AstraZeneca, Dracen Pharmaceuticals, and WindMIL; he is a scientific advisor/consultant for AstraZeneca, Bristol-Myers Squibb, Leica Biosystems, Jazz Pharmaceuticals, Novocure, Pfizer, and MorphoSys; and his spouse is employed by and owns stock in Boston Scientific. The remaining authors have disclosed that they have no financial interests, arrangement affiliations, or commercial interests with the manufacturers of any products discussed in this article or their competitors.

Correspondence: Aaron E. Lisberg, MD, 2020 Santa Monica Boulevard, Suite 600, Santa Monica, CA 90404. Email: alisberg@mednet.ucla.edu

\section{References}

1. Borghaei $H$, Paz-Ares $L$, Horn L, et al. Nivolumab versus docetaxel in advanced nonsquamous non-small cell lung cancer. N Engl J Med 2015;373:1627-1639.

2. Brahmer J, Reckamp KL, Baas $P$, et al. Nivolumab versus docetaxel in advanced squamous-cell nonsmall-cell lung cancer. N Engl J Med 2015:373:123-135.

3. Reck M, Rodríguez-Abreu D, Robinson AG, et al. Pembrolizumab versus chemotherapy for PD-L1positive non-small-cell lung cancer. N Engl J Med 2016;375:1823-1833.

4. Weber JS, Postow M, Lao CD, Schadendorf D. Management of adverse events following treatment with anti-programmed death-1 agents. Oncologist 2016;21:1230-1240.

5. Kichenadasse G, Miners JO, Mangoni AA, et al. Multiorgan immune-related adverse events during treatment with atezolizumab. J Natl Compr Canc Netw 2020;18:1191-1199.

6. Rittmeyer A, Barlesi F, Waterkamp D, et al. Atezolizumab versus docetaxel in patients with previously treated non-small-cell lung cancer (OAK): a phase 3, open-label, multicentre randomised controlled trial. Lancet 2017;389:255-265.

7. Fehrenbacher L, Spira A, Ballinger M, et al. Atezolizumab versus docetaxel for patients with previously treated non-small-cell lung cancer (POPLAR): a multicentre, open-label, phase 2 randomised controlled trial. Lancet 2016;387:1837-1846.

8. Peters $\mathrm{S}$, Gettinger S, Johnson ML, et al. Phase II trial of atezolizumab as first-line or subsequent therapy for patients with programmed death-ligand 1-selected advanced non-small-cell lung cancer (BIRCH). J Clin Oncol 2017;35:2781-2789.

9. Spigel DR, Chaft JE, Gettinger S, et al. FIR: Efficacy, safety, and biomarker analysis of a phase II open-labe study of atezolizumab in PD-L1-selected patients with NSCLC. J Thorac Oncol 2018;13:1733-1742.

10. Dupont $R$, Bérard $E$, Puisset $F$, et al. The prognostic impact of immune-related adverse events during anti-PD1 treatment in melanoma and non-small-cell lung cancer: a real-life retrospective study [published online November 5, 2019]. Oncolmmunology, doi:10.1080/2162402X.2019.1682383

11. Kartolo A, Sattar J, Sahai V, et al. Predictors of immunotherapy-induced immune-related adverse events [published online November 2, 2018]. Curr Oncol, doi:10.3747/co.25.4047

12. Cortellini A, Chiari R, Ricciuti $B$, et al. Correlations between the immune-related adverse events spectrum and efficacy of anti-PD1 immunotherapy in NSCLC patients. Clin Lung Cancer 2019;20: 237-247.

13. Nigro O, Cortellini A, Giusti R, et al. Incidence and clinical implications of late immune-related adverse events in long responders to PD-1/PD-L1 checkpoint inhibitors: a multicenter study. Ann Oncol 2019 30 (Suppl 11). doi:10.1093/annonc/mdz449.009

14. Okada N, Kawazoe H, Takechi K, et al. Association between immune-related adverse events and clinical efficacy in patients with melanoma treated with nivolumab: a multicenter retrospective study. Clin Ther 2019:41:Abstract X120.

15. Singh $P$, Brito A, Abdulnour REE, et al. Defining real-world criteria for immune-related adverse events (irAEs) [abstract]. J Clin Oncol 2019;37(Suppl):Abstract e14172.

16. Abu-Sbeih $\mathrm{H}$, Tang $\mathrm{T}$, Ali FS, et al. The impact of immune checkpoint inhibitor-related adverse events and their immunosuppressive treatment on patients' outcomes. J Immunother Precis Oncol 2018;1: 7-18.

17. Ricciuti B, Genova C, De Giglio A et al. Impact of immune-related adverse events on survival in patients with advanced non-small cell lung cancer treated with nivolumab: long-term outcomes from a multiinstitutional analysis. J Cancer Res Clin Oncol 2019;145:479-485.

18. Le Burel S, Champiat S, Mateus $C$, et al. Prevalence of immune-related systemic adverse events in patients treated with anti-programmed cell death 1/anti-programmed cell death-ligand 1 agents: a single-centre pharmacovigilance database analysis. Eur J Cancer 2017;82:34-44

19. Owen DH, Wei L, Bertino EM, et al. Incidence, risk factors, and effect on survival of immune-related adverse events in patients with non-small-cell lung cancer. Clin Lung Cancer 2018;19:e893-900.

20. Lisberg A, Tucker DA, Goldman JW, et al. Treatment related adverse events predict improved clinical outcome in NSCLC patients on KEYNOTE-001 at a single center [published online January 1, 2018]. Cancer Immunol Res, doi:10.1158/2326-6066.CIR-17-0063

21. von Itzstein MS, Khan S, Gerber DE. Investigational biomarkers for checkpoint inhibitor immune-related adverse event prediction and diagnosis. Clin Chem 2020;66:779-793.

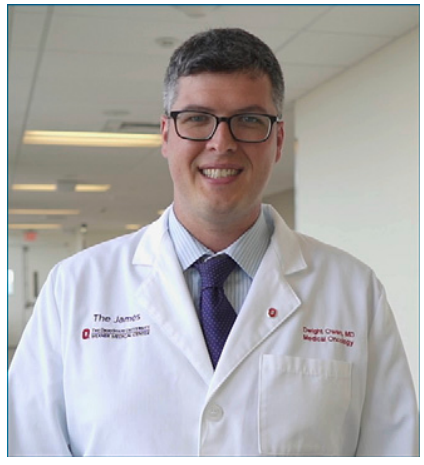

DWIGHT OWEN, MD

Dwight Owen, MD, is Assistant Professor of Internal Medicine, Division of Medical Oncology, at Ohio State University. His research focuses on developing novel therapeutic strategies for the treatment of thoracic malignancies utilizing immunotherapy and targeted agents. He has created a database of immune-related adverse events at Ohio State University that has allowed for multidisciplinary collaboration with faculty in other disease areas to better characterize the incidence of immunerelated adverse events as well as optimal management. He has been awarded multiple professional honors and has published extensively. 


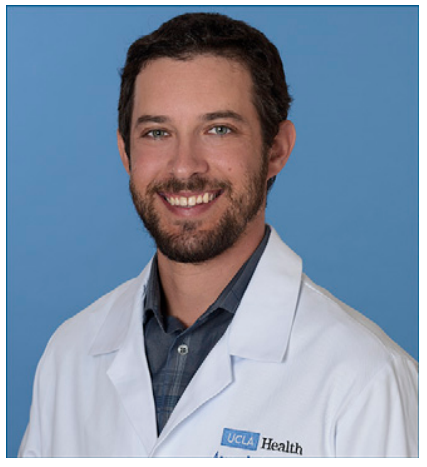

\section{AARON E. LISBERG, MD}

Aaron E. Lisberg, MD, is a medical oncologist with an emphasis on clinical and translational research in thoracic oncology at the David Geffen School of Medicine at UCLA. He conducts translational research aimed at identifying novel therapeutic approaches for the treatment of thoracic malignancies, with a specific focus on immune-based and metabolically targeted therapies and has published extensively.
22. Khoja L, Day D, Wei-Wu Chen T, et al. Tumour- and class-specific patterns of immune-related adverse events of immune checkpoint inhibitors: a systematic review. Ann Oncol 2017;28:2377-2385.

23. Stroud CR, Hegde A, Cherry C, et al. Tocilizumab for the management of immune mediated adverse events secondary to PD-1 blockade. J Oncol Pharm Pract 2019;25:551-557.

24. Das S, Johnson DB. Immune-related adverse events and anti-tumor efficacy of immune checkpoint inhibitors [published November 15,2019]. J Immunother Cancer, doi:10.1186/s40425-019-0805-8

25. Gandhi L, Rodríguez-Abreu D, Gadgeel S, et al. Pembrolizumab plus chemotherapy in metastatic non-small-cell lung cancer. N Engl J Med 2018;378:2078-2092.

26. Antonia SJ, Villegas A, Daniel D, et al. Durvalumab after chemoradiotherapy in stage III non-small-cell lung cancer. N Engl J Med 2017;377:1919-1929.

27. Rini BI, Plimack ER, Stus V, et al. Pembrolizumab plus axitinib versus sunitinib for advanced renal-cell carcinoma. N Engl J Med 2019;380:1116-1127.

28. Brahmer JR, Lacchetti C, Schneider BJ, et al. Management of immune-related adverse events in patients treated with immune checkpoint inhibitor therapy: American Society of Clinical Oncology Clinical Practice Guideline. J Clin Oncol 2018;36:1714-1768.

29. Thompson JA, Schneider BJ, Brahmer JR, et al. NCCN Clinical Practice Guidelines in Oncology: Management of Immunotherapy-Related Toxicities, Version 1.2020. Accessed August 7, 2020. To view the most recent version, visit NCCN.org.

30. Davis EJ, Salem JE, Young A, et al. Hematologic complications of immune checkpoint inhibitors. Oncologist 2019;24:584-588. 